\title{
The Butterfly Effect
}

\author{
Étienne Ghys
}

\begin{abstract}
It is very unusual for a mathematical idea to disseminate into the society at large. An interesting example is chaos theory, popularized by Lorenz's butterfly effect: "does the flap of a butterfly's wings in Brazil set off a tornado in Texas?" A tiny cause can generate big consequences! Can one adequately summarize chaos theory in such a simple minded way? Are mathematicians responsible for the inadequate transmission of their theories outside of their own community? What is the precise message that Lorenz wanted to convey? Some of the main characters of the history of chaos were indeed concerned with the problem of communicating their ideas to other scientists or non-scientists. I'll try to discuss their successes and failures. The education of future mathematicians should include specific training to teach them how to explain mathematics outside their community. This is more and more necessary due to the increasing complexity of mathematics. A necessity and a challenge!
\end{abstract}

\section{Introduction}

In 1972, the meteorologist Edward Lorenz gave a talk at the 139th meeting of the American Association for the Advancement of Science entitled "Does the flap of a butterfly's wings in Brazil set off a tornado in Texas?". Forty years later, a google search "butterfly effect" generates ten million answers. Surprisingly most answers are not related to mathematics or physics and one can find the most improbable websites related to movies, music, popular books, video games, religion, philosophy and even Marxism! It is very unusual that a mathematical idea can disseminate into the general society. One could mention Thom's catastrophe theory in the 1970s, or Mandelbrot's fractals in the 1980s, but these theories remained confined to the scientifically oriented population. On the contrary, chaos theory, often

\footnotetext{
É. Ghys $(\square)$

CNRS-UMPA ENS Lyon, Lyon, France

e-mail: etienne.ghys@ens-lyon.fr

(C) The Author(s) 2015

S.J. Cho (ed.), The Proceedings of the 12th International Congress

on Mathematical Education, DOI 10.1007/978-3-319-12688-3_6
} 
presented through the butterfly effect, did penetrate the nonscientific population at a very large scale. Unfortunately, this wide diffusion was accompanied with an oversimplification of the main original ideas and one has to admit that the transmission procedure from scientists to nonscientists was a failure. As an example, the successful book The butterfly effect by Andy Andrews "reveals the secret of how you can live a life of permanent purpose" and "shows how your everyday actions can make a difference for generations to come" which is not exactly the message of the founding fathers of chaos theory! In Spielberg's movie Jurassic Park, Jeff Goldblum introduces himself as a "chaotician" and tries (unsuccessfully) to explain the butterfly effect and unpredictability to the charming Laura Dern; the message is scientifically more accurate but misses the main point. If chaos theory only claimed that the future is unpredictable, would it deserve the name "theory"? After all, it is well known that "Prediction is very difficult, especially the future!". ${ }^{2}$ A scientific theory cannot be limited to negative statements and one would be disappointed if Lorenz's message only contained this well known fact.

The purpose of this talk is twofold. On the one hand, I would like to give a very elementary presentation of chaos theory, as a mathematical theory, and to give some general overview on the current research activity in this domain with an emphasis on the role of the so-called physical measures. On the other hand, I would like to analyze the historical process of the development of the theory, its successes and failures, focusing in particular on the transmission of ideas between mathematics and physics, or from Science to the general public. This case study might give us some hints to improve the communication of mathematical ideas outside mathematics or scientific circles. The gap between mathematicians and the general population has never been so wide. This may be due to the increasing complexity of mathematics or to the decreasing interest of the population for Science. I believe that the mathematical community has the responsibility of building bridges.

\section{A Brief History of Chaos from Newton to Lorenz}

\section{Determinism}

One of the main pillars of Science is determinism: the possibility of prediction. This is of course not due to a single person but one should probably emphasize the fundamental role of Newton. As he was laying the foundations of differential calculus and unraveling the laws of mechanics, he was offering by the same token a tool enabling predictions. Given a mechanical system, be it the solar system or the collection of molecules in my room, one can write down a differential equation governing the motion. If one knows the present position and velocity of the system, one should

\footnotetext{
1 See www.peterpatau.com/2006/12/bohr-leads-berra-but-yogi-closing-gap.html for an interesting discussion of the origin of this quotation.
} 
simply solve a differential equation in order to determine the future. Of course, solving a differential equation is not always a simple matter but this implies at least the principle of determinism: the present situation determines the future. Laplace summarized this wonderfully in his "Essai philosophique sur les probabilités" (Laplace, 1814):

We ought then to consider the present state of the universe as the effect of its previous state and as the cause of that which is to follow. An intelligence that, at a given instant, could comprehend all the forces by which nature is animated and the respective situation of the beings that make it up, if moreover it were vast enough to submit these data to analysis, would encompass in the same formula the movements of the greatest bodies of the universe and those of the lightest atoms. For such an intelligence nothing would be uncertain, and the future, like the past, would be open to its eyes.

The fact that this quotation comes from a book on probability theory shows that Laplace's view on determinism was far from naïve (Kahane 2008). We lack the "vast intelligence" and we are forced to use probabilities in order to understand dynamical systems.

\section{Sensitivity to Initial Conditions}

In his little book "Matter and Motion", Maxwell insists on the sensitivity to initial conditions in physical phenomena (Maxwell, 1876):

There is a maxim which is often quoted, that 'The same causes will always produce the same effects.' To make this maxim intelligible we must define what we mean by the same causes and the same effects, since it is manifest that no event ever happens more that once, so that the causes and effects cannot be the same in all respects. [...]

There is another maxim which must not be confounded with that quoted at the beginning of this article, which asserts 'That like causes produce like effects'. This is only true when small variations in the initial circumstances produce only small variations in the final state of the system. In a great many physical phenomena this condition is satisfied; but there are other cases in which a small initial variation may produce a great change in the final state of the system, as when the displacement of the 'points' causes a railway train to run into another instead of keeping its proper course.

Notice that Maxwell seems to believe that "in great many cases" there is no sensitivity to initial conditions. The question of the frequency of chaos in nature is still at the heart of current research. Note also that Maxwell did not really describe what we would call chaos today. Indeed, if one drops a rock from the top of a mountain, it is clear that the valley where it will end its course can be sensitive to a small variation of the initial position but it is equally clear that the motion cannot be called "chaotic" in any sense of the word: the rock simply goes downwards and eventually stops. 


\title{
Fear for Chaos
}

It is usually asserted that chaos was "discovered" by Poincaré in his famous memoir on the 3-body problem (Poincaré 1890). His role is without doubt very important, but maybe not as much as is often claimed. He was not the first to discover sensitivity to initial conditions. However, he certainly realized that some mechanical motions are very intricate, in a way that Maxwell had not imagined. Nevertheless chaos theory cannot be limited to the statement that the dynamics is complicated: any reasonable theory must provide methods allowing some kind of understanding. The following famous quotation of Poincaré illustrates his despair when confronted by the complication of dynamics (Poincaré 1890):

\begin{abstract}
When we try to represent the figure formed by these two curves and their infinitely many intersections, each corresponding to a doubly asymptotic solution, these intersections form a type of trellis, tissue, or grid with infinitely fine mesh. Neither of the two curves must ever cut across itself again, but it must bend back upon itself in a very complex manner in order to cut across all of the meshes in the grid an infinite number of times. The complexity of this figure is striking, and I shall not even try to draw it. Nothing is more suitable for providing us with an idea of the complex nature of the three-body problem, and of all the problems of dynamics in general [...].
\end{abstract}

One should mention that ten years earlier Poincaré had written a fundamental memoir "Sur les courbes définies par des équations différentielles" laying the foundations of the qualitative theory of dynamical systems (Poincaré 1881). In this paper, he had analyzed in great detail the behavior of the trajectories of a vector field in the plane, i.e. of the solutions of an ordinary differential equation in dimension 2. One of his main results - the Poincaré-Bendixson theorem-implied that such trajectories are very well behaved and converge to an equilibrium point or to a periodic trajectory (or to a so-called "graphic"): nothing chaotic in dimension 2! In his 1890 paper, he was dealing with differential equations in dimension 3 and he must have been puzzled - and scared - when he realized the complexity of the picture.

\section{Taming Chaos}

Hadamard wrote a fundamental paper on the dynamical behavior of geodesics on negatively curved surfaces (Hadamard, 1898). He first observes that "a tiny change of direction of a geodesic [...] is sufficient to cause any variation of the final shape of the curve" but he goes much further and creates the main concepts of the so-called "symbolic dynamics". This enables him to prove positive statements, giving a fairly precise description of the behavior of geodesics. Of course, Hadamard is perfectly aware of the fact that geodesics on a surface define a very primitive mechanical system and that it is not clear at all that natural phenomena could have a similar behavior. He concludes his paper in a cautious way: 
Will the circumstances we have just described occur in other problems of mechanics? In particular, will they appear in the motion of celestial bodies? We are unable to make such an assertion. However, it is likely that the results obtained for these difficult cases will be analogous to the preceding ones, at least in their degree of complexity. [...]

Certainly, if a system moves under the action of given forces and its initial conditions have given values in the mathematical sense, its future motion and behavior are exactly known. But, in astronomical problems, the situation is quite different: the constants defining the motion are only physically known, that is with some errors; their sizes get reduced along the progresses of our observing devices, but these errors can never completely vanish.

So far, the idea that some physical systems could be complicated and sensitive to small variations of the initial conditions - making predictions impossible in practice - remained hidden in very confidential mathematical papers known to a very small number of scientists. One should keep in mind that by the turn of the century, physics was triumphant and the general opinion was that Science would eventually explain everything. The revolutionary idea that there is a strong conceptual limitation to predictability was simply unacceptable to most scientists.

\section{Popularization}

However, at least two scientists realized that this idea is relevant in Science and tried - unsuccessfully - to advertize it outside mathematics and physics, in "popular books".

In his widely circulated book Science and Method, Poincaré expresses the dependence to initial conditions in a very clear way. The formulation is very close to the butterfly slogan and even includes a devastating cyclone (Poincaré 1908):

Why have meteorologists such difficulty in predicting the weather with any certainty? Why
is it that showers and even storms seem to come by chance, so that many people think it
quite natural to pray for rain or fine weather, though they would consider it ridiculous to ask
for an eclipse by prayer? We see that great disturbances are generally produced in regions
where the atmosphere is in unstable equilibrium. The meteorologists see very well that the
equilibrium is unstable, that a cyclone will be formed somewhere, but exactly where they
are not in a position to say; a tenth of a degree more or less at any given point, and the
cyclone will burst here and not there, and extend its ravages over districts it would
otherwise have spared. If they had been aware of this tenth of a degree they could have
known it beforehand, but the observations were neither sufficiently comprehensive nor
sufficiently precise, and that is the reason why it all seems due to the intervention of chance.

In 1908 Poincaré was less scared by chaos than in 1890. He was no longer considering chaos as an obstacle to a global understanding of the dynamics, at least from the probabilistic viewpoint. Reading Poincaré's papers of this period, with today's understanding of the theory, one realizes that he had indeed discovered the role of what is called today physical measures (to be discussed later) which are at the heart of the current approach. Unfortunately, none of his contemporaries could grasp the idea - or maybe he did not formulate it in a suitable way — and one had to wait for seventy years before the idea could be re-discovered! 
You are asking me to predict future phenomena. If, quite unluckily, I happened to know the laws of these phenomena, I could achieve this goal only at the price of inextricable computations, and should renounce to answer you; but since I am lucky enough to ignore these laws, I will answer you straight away. And the most astonishing is that my answer will be correct.

Another attempt to advertize these ideas outside mathematics and physics was made by Duhem (1906) in his book The aim and structure of physical theory. His purpose was to popularize Hadamard's paper and he used simple words and very efficient "slogans":

Imagine the forehead of a bull, with the protuberances from which the horns and ears start, and with the collars hollowed out between these protuberances; but elongate these horns and ears without limit so that they extend to infinity; then you will have one of the surfaces we wish to study. On such a surface geodesics may show many different aspects. There are, first of all, geodesics which close on themselves. There are some also which are never infinitely distant from their starting point even though they never exactly pass through it again; some turn continually around the right horn, others around the left horn, or right ear, or left ear; others, more complicated, alternate, in accordance with certain rules, the turns they describe around one horn with the turns they describe around the other horn, or around one of the ears. Finally, on the forehead of our bull with his unlimited horns and ears there will be geodesics going to infinity, some mounting the right horn, others mounting the left horn, and still others following the right or left ear. [...] If, therefore, a material point is thrown on the surface studied starting from a geometrically given position with a geometrically given velocity, mathematical deduction can determine the trajectory of this point and tell whether this path goes to infinity or not. But, for the physicist, this deduction is forever unutilizable. When, indeed, the data are no longer known geometrically, but are determined by physical procedures as precise as we may suppose, the question put remains and will always remain unanswered.

Unfortunately the time was not ripe. Scientists were not ready for the message... Poincaré and Duhem were not heard. The theory went into a coma. Not completely though, since Birkhoff continued the work of Poincaré in a strictly mathematical way, with no attempts to develop a school, and with no applications to natural sciences. One should mention that Poincaré's work had also some posterity in the Soviet Union but this was more related to the 1881 "non chaotic" theory of limit cycles (Aubin and Dahan Dalmedico 2002).

Later I will describe Lorenz's fundamental article which bears the technical title "Deterministic non periodic flow", and was largely unnoticed by mathematicians for about ten years (Lorenz, 1963). Lorenz gave a lecture entitled "Predictability: does the flap of a butterfly's wings in Brazil set off a tornado in Texas?" which was the starting point of the famous butterfly effect (Lorenz, 1972).

If a single flap of a butterfly's wing can be instrumental in generating a tornado, so all the previous and subsequent flaps of its wings, as can the flaps of the wings of the millions of other butterflies, not to mention the activities of innumerable more powerful creatures, including our own species.

If a flap of a butterfly's wing can be instrumental in generating a tornado, it can equally well be instrumental in preventing a tornado. 
This is not really different from Poincaré's "a tenth of a degree more or less at any given point, and the cyclone will burst here and not there". However, meanwhile, physics (and mathematics) had gone through several revolutions and non-predictability had become an acceptable idea. More importantly, the world had also gone through several (more important) revolutions. The message "each one of us can change the world" was received as a sign of individual freedom. This is probably the explanation of the success of the butterfly effect in popular culture. It would be interesting to describe how Lorenz's talk reached the general population. One should certainly mention the best seller Chaos: making a new science (Gleick 1987) (which was a finalist for the Pulitzer Prize). One should not minimize the importance of such books. One should also emphasize that Lorenz himself published a wonderful popular book The essence of chaos in 1993. Note that the two main characters of the theory, Poincaré and Lorenz, wrote popular books to make their researches accessible to a wide audience.

\section{Lorenz's 1963 Paper}

Lorenz's article is wonderful (Lorenz 1963). At first unnoticed, it eventually became one of the most cited papers in scientific literature (more than 6,000 citations since 1963 and about 400 each year in recent years). For a few years, Lorenz had been studying simplified models describing the motion of the atmosphere in terms of ordinary differential equations depending on a small number of variables. For instance, in 1960 he had described a system that can be explicitly solved using elliptic functions: solutions were quasiperiodic in time (Lorenz 1960). His article (Lorenz 1962) analyzes a differential equation in a space of dimension 12, in which he numerically detects a sensitive dependence to initial conditions. His 1963 paper lead him to fame.

In this study we shall work with systems of deterministic equations which are idealizations of hydrodynamical systems.

After all, the atmosphere is made of finitely many particles, so one indeed needs to solve an ordinary differential equation in a huge dimensional space. Of course, such equations are intractable, and one must treat them as partial differential equations. In turn, the latter must be discretized on a finite grid, leading to new ordinary differential equations depending on fewer variables, and probably more useful than the original ones.

The bibliography in Lorenz's article includes one article of Poincaré, but not the right one! He cites the early 1881 "non chaotic" memoir dealing with 2 dimensional dynamics. Lorenz seems indeed to have overlooked the Poincaré's papers that we have discussed above. Another bibliographic reference is a book by Birkhoff (1927)

2 Subtitle of a book by Bill Clinton (2007). 
on dynamical systems. Again, this is not "the right" reference since the "significant" papers on chaos by Birkhoff were published later. On the occasion of the 1991 Kyoto prize, Lorenz gave a lecture entitled "A scientist by choice" in which he discusses his relationship with mathematics (Lorenz 1991). In 1938 he was a graduate student in Harvard and was working under the guidance of... Birkhoff "on a problem in mathematical physics". However he seems unaware of the fact that Birkhoff was indeed the best follower of Poincaré. A missed opportunity? On the other hand, Lorenz mentions that Birkhoff "was noted for having formulated a theory of aesthetics".

Lorenz considers the phenomenon of convection. A thin layer of a viscous fluid is placed between two horizontal planes, set at two different temperatures, and one wants to describe the resulting motion. The higher parts of the fluid are colder, therefore denser; they have thus a tendency to go down due to gravity, and are then heated when they reach the lower regions. The resulting circulation of the fluid is complex. Physicists are very familiar with the Bénard and Rayleigh experiments. Assuming the solutions are periodic in space, expanding in Fourier series and truncating these series to keep only a small number of terms, Salzman had just obtained an ordinary differential equation describing the evolution. Drastically simplifying this equation, Lorenz obtained "his" differential equation:

$$
\frac{d x}{d t}=\sigma(x+y) ; \frac{d y}{d t}=-x z+r z-y ; \frac{d z}{d t}=x y-b z
$$

Here $x$ represents the intensity of the convection, $y$ represents the temperature difference between the ascending and descending currents, and $z$ is proportional to the "distortion" of the vertical temperature profile from linearity, a positive value indicating that the strongest gradients occur near the boundaries. Obviously, one should not seek in this equation a faithful representation of the physical phenomenon. The constant $\sigma$ is the Prandtl number. Guided by physical considerations, Lorenz was lead to choose the numerical values $r=28, \sigma=10, b=8 / 3$. It was a good choice, and these values remain traditional today. He could then numerically solve these equations, and observe a few trajectories. The electronic computer Royal McBee LGP-30 was rather primitive: according to Lorenz, it computed (only!) 1,000 times faster than by hand. The anecdote is well known (Lorenz 1991):

I started the computer again and went out for a cup of coffee. When I returned about an hour later, after the computer had generated about two months of data, I found that the new solution did not agree with the original one. [...] I realized that if the real atmosphere behaved in the same manner as the model, long-range weather prediction would be impossible, since most real weather elements were certainly not measured accurately to three decimal places.

Let us introduce some basic terminology and notation. For simplicity we shall only deal with ordinary differential equations in $\mathbb{R}^{n}$ of the form $\frac{d x}{d t}=X(x)$ where $x$ is now a point in $\mathbb{R}^{n}$ and $X$ is a vector field in $\mathbb{R}^{n}$. We shall assume that $X$ is transversal to some large sphere, say $\|x\|=R$, pointing inwards, which means that the scalar 
product $x . X(x)$ is negative on this sphere. Denote by $B$ the ball $\|x\| \leq R$. For any point $x$ in $B$, there is a unique solution of the differential equation with initial condition $x$ and defined for all $t \geq 0$. Denote this solution by $\phi^{t}(x)$. The purpose of the theory of dynamical systems is to understand the asymptotic behavior of these trajectories when $t$ tends to infinity. With this terminology, one says that $X$ is sensitive to initial conditions if there exists some $\delta>0$ such that for every $\epsilon>0$ one can find two points $x, x^{\prime}$ in $B$ with $\left\|x-x^{\prime}\right\|<\epsilon$ and some time $t>0$ such that $\left\|\phi^{t}(x)-\phi^{t}\left(x^{\prime}\right)\right\|<\delta$.

Lorenz's observations go much further than the fact that "his" differential equation is sensitive to initial conditions. He notices that these unstable trajectories seem to accumulate on a complicated compact set, which is itself insensitive to initial conditions and he describes this limit set in a remarkably precise way. There exists some compact set $K$ in the ball such that for almost every initial condition $x$, the trajectory of $x$ accumulates precisely on $K$. This attracting set $K$ (now called the Lorenz attractor) approximately resembles a surface presenting a "double" line along which two leaves merge.

Thus within the limits of accuracy of the printed values, the trajectory is confined to a pair of surfaces which appear to merge in the lower portion. [...] It would seem, then, that the two surfaces merely appear to merge, and remain distinct surfaces. [...] Continuing this process for another circuit, we see that there are really eight surfaces, etc., and we finally conclude that there is an infinite complex of surfaces, each extremely close to one or the other of the two merging surfaces.

Lorenz (1963)

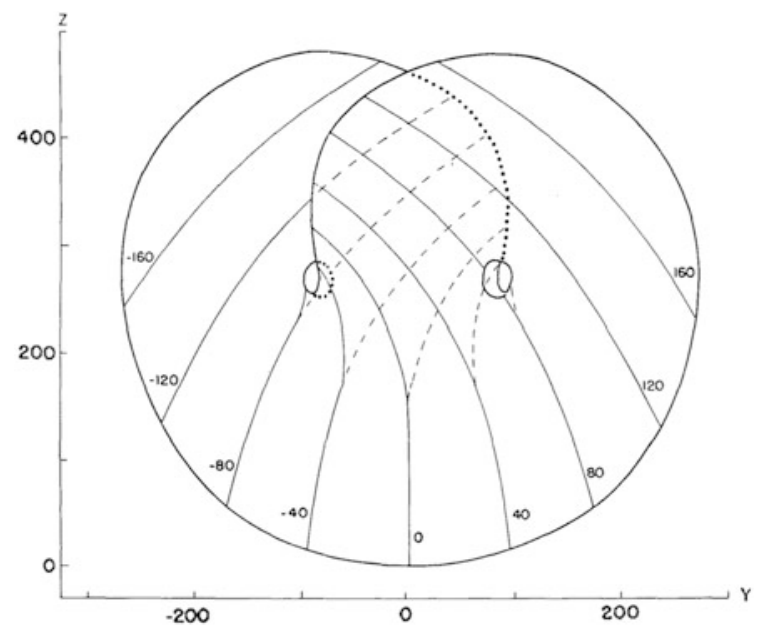

Starting from an initial condition, the trajectory rapidly approaches this "two dimensional object" and then travels "on" this "surface". The trajectory turns around the two holes, left or right, in a seemingly random way. Notice the analogy with Hadamard's geodesics turning around the horns of a bull. Besides, Lorenz 
studies how trajectories come back to the "branching line" where the two surfaces merge, which can be parameterized by some interval $[0,1]$. Obviously, this interval is not very well defined, since the two merging surfaces do not really come in contact, although they coincide "within the limits of accuracy of the printed values". Starting from a point on this "interval", one can follow the future trajectory and observe its first return onto the interval. This defines a two to one map from the interval to itself. Indeed, in order to go back in time and track the past trajectory of a point in $[0,1]$, one should be able to select one of the two surfaces attached to the interval. On the figure the two different past trajectories seem to emanate from the "same point" of the interval. Of course, if there are two past trajectories starting from "one" point, there should be four, then eight, etc., which is what Lorenz expresses in the above quotation. Numerically, the first return map is featured on the left part of Figure, extracted from the original paper.
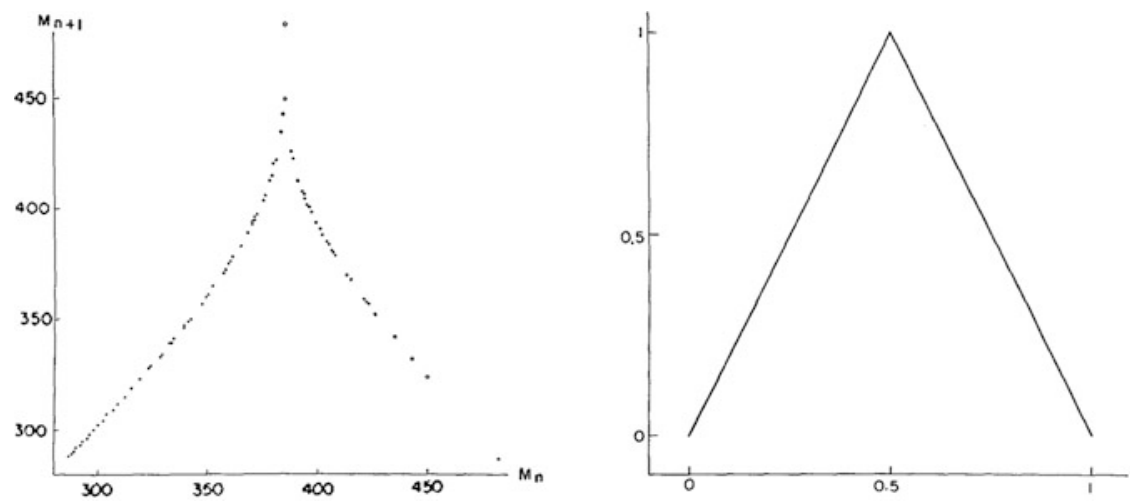

Working by analogy, Lorenz compares this map to the (much simpler) following one: $f(x)=2 x$ if $0 \leq x \leq \frac{1}{2}$ and $f(x)=2-2 x$ if $\frac{1}{2} \leq x \leq 1$ (right part of the Figure). Nowadays the chaotic behavior of this "tent map" is well known, but this was much less classical in 1963. In particular, the periodic points of $f$ are exactly the rational numbers with odd denominators, which are dense in $[0,1]$. Lorenz does not hesitate to claim that the same property applies to the iterations of the "true" return map. The periodic trajectories of the Lorenz attractor are "therefore" dense in $K$. What an intuition! Finally, he concludes with a lucid question on the relevance of his model for the atmosphere.

There remains the question as to whether our results really apply to the atmosphere. One does not usually regard the atmosphere as either deterministic or finite, and the lack of periodicity is not a mathematical certainty, since the atmosphere has not been observed forever.

To summarize, this remarkable article contains the first example of a physically relevant dynamical system presenting all the characteristics of chaos. Individual trajectories are unstable but their asymptotic behavior seems to be insensitive to 
initial conditions: they converge to the same attractor. None of the above assertions are justified, at least in the mathematical sense. How frustrating!

Surprisingly, an important question is not addressed in Lorenz's article. The observed behavior happens to be robust: if one slightly perturbs the differential equation, for instance by modifying the values of the parameters, or by adding small terms, then the new differential equation will feature the same type of attractor with the general aspect of a branched surface. This property would be rigorously established much later by Guckhenheimer and Williams.

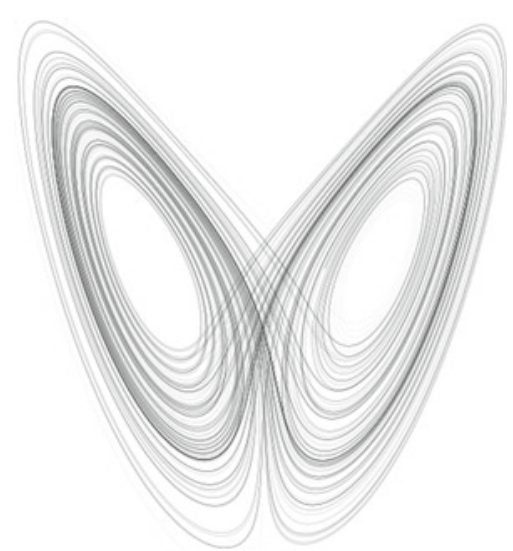

The Lorenz attractor looks like a butterfly

\section{Meanwhile, Mathematicians...}

\section{Lack of Communication Between Mathematicians and Physicists?}

Mathematicians did not notice Lorenz's paper for more than ten years. The mathematical activity in dynamical systems during this period followed an independent and parallel path, under the lead of Smale. How can one understand this lack of communication between Lorenz - the MIT meteorologist - and Smale - the Berkeley mathematician? Obviously, during the 1960s the scientific community had already reached such a size that it was impossible for a single person to master mathematics and physics; the time of Poincaré was over. No bridge between different sciences was available. Mathematicians had no access to the Journal of Atmospheric Sciences. ${ }^{3}$

\footnotetext{
3 In order to find an excuse for not having noticed Lorenz paper, a famous mathematician told me that Lorenz had published in "some obscure journal"!.
} 


\section{Smale's Axiom A}

In 1959 Smale had obtained remarkable results in topology, around the Poincaré conjecture in higher dimension. The main tool was Morse theory describing the gradient of a (generic) function. The dynamics of such a gradient is far from chaotic: trajectories go uphill and converge to some equilibrium point. Smale initiated a grandiose program aiming at a qualitative description of the trajectories of a generic vector field (on compact manifolds). His first attempt was amazingly naïve (Smale 1960). He conjectured that a generic vector field has a finite number of equilibrium points, a finite number of periodic trajectories, and that every trajectory converges in the future (and in the past) towards an equilibrium or a periodic trajectory. He was therefore proposing that chaos does not exist! Poincaré, Hadamard or Birkhoff had already published counterexamples many years earlier! Looking back at this period, Smale wrote (1998a, b):

It is astounding how important scientific ideas can get lost, even when they are aired by leading scientific mathematicians of the preceding decades.

Smale realized soon by himself ${ }^{4}$ that the dynamics of a generic vector field is likely to be much more complicated than he had expected. He constructed a counterexample to his own conjecture (Smale 1961). The famous horseshoe is a simple example of a dynamical system admitting an infinite number of periodic trajectories in a stable way.

In order to describe this example, I should explain a classical construction (due to Poincaré). Suppose we start with a vector field $X$ (in a ball in $\mathbb{R}^{n}$, as above). It may happen that one can find some $n-1$ dimensional disc $D$, which is transverse to $X$ and which is such that the trajectory of every point $x$ in $D$ intersects $D$ infinitely often. In such a situation, one can define a map $F: D \rightarrow D$ which associates to each point $x$ in $D$ the next intersection of its trajectory with $D$. For obvious reasons, this map is called the first return map. Clearly the description of the dynamics of $X$ reduces to the description of the iterates of $F$. Conversely, in many cases, one can construct a vector field from a map $F$. It is often easier to draw pictures in $D$ since it is one dimension lower than $B$. In Smale's example, $D$ has dimension 2 and corresponds to a vector field in dimension 3, like in Lorenz's example. The map $F$ is called a horseshoe map since the image $F(C)$ of a square $C$ does look like a horseshoe as in the picture.

\footnotetext{
4 As if obeying Goethe's dictum "Was du ererbt von deinen Vätern hast, erwirb es, um es zu besitzen" ("That which you have inherited from your fathers, earn it in order to possess it.").
} 


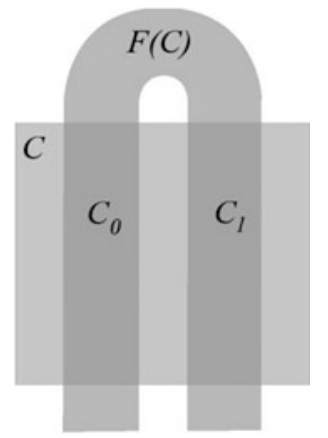

The infinite intersection $\cap_{-\infty}^{+\infty} F^{i}(C)$ is a nonempty compact set $K \subset D$, and the restriction of $F$ to $K$ is a homeomorphism. The intersection $C \cap F(C)$ consists of two connected components $C_{0}$ and $C_{1}$. Smale shows that one can choose $F$ in such a way that for every bi-infinite sequence $a_{i}$ (with $a_{i}=0$ or 1 ), there exists a unique point $x$ in $K$ such that $F^{i}(x) \in C_{i}$ for every $i$. In particular, periodic points of $F$ correspond to periodic sequences $a_{i}$; they are dense in $K$.

More importantly, Smale shows that his example is structurally stable. Let us come back to a vector field $X$ defined in some ball in $\mathbb{R}^{n}$ and transversal to the boundary. One says that $X$ is structurally stable if every vector field $X^{\prime}$ which is close enough to $X$ (say in the $C^{1}$ topology) is topologically conjugate to $X$ : there is a homeomorphism $h$ of $B$ sending trajectories of $X$ to trajectories of $X^{\prime}$. Andronov and Pontryagin (1937) had introduced this concept in 1937 but in a very simple context, certainly not in the presence of an infinite number of periodic trajectories. The proof that the horseshoe map defines a structurally stable vector field is rather elementary. It is based on the fact that a map $F^{\prime}$ from $D$ to itself close enough to $F$ is also described by the same infinite sequences $a_{i}$.

Smale published this result in the proceedings of a workshop organized in the Soviet Union in 1961. Anosov tells us about this "revolution" in Anosov (2006).

The world turned upside down for me, and a new life began, having read Smale's announcement of 'a structurally stable homeomorphism with an infinite number of periodic points', while standing in line to register for a conference in Kiev in 1961. The article is written in a lively, witty, and often jocular style and is full of captivating observations. [...] [Smale] felt like a god who is to create a universe in which certain phenomena would occur.

Afterwards the theory progressed at a fast pace. Smale quickly generalized the horseshoe; see for instance (Smale 1966). Anosov proved in 1962 that the geodesic flow on a manifold of negative curvature is structurally stable (Anosov 1962) ${ }^{5}$. For this purpose, he created the concept of what is known today as Anosov flows. Starting from the known examples of structurally stable systems, Smale cooked up in 1965 the fundamental concept of dynamical systems satisfying the Axiom A and conjectured that these systems are generic and structurally stable. Smale's (1967) 5 Surprisingly, he does not seem to be aware of Hadamard's work. It would not be difficult to
deduce Anosov's theorem from Hadamard's paper. 
article "Differential dynamical systems" represents an important step for the theory of dynamical systems (Smale 1967), a "masterpiece of mathematical literature" according to Ruelle. But, already in 1966, Abraham and Smale found a counterexample to this second conjecture of Smale: Axiom A systems are indeed structurally stable but they are not generic (Smale 1966, Abraham and Smale 1968).

\section{Lorenz's Equation Enters the Scene}

Lorenz's equation pops up in mathematics in the middle of the 1970s. According to Guckenheimer, Yorke mentioned to Smale and his students the existence of Lorenz's equation, which did not fit well with their approach. The well-known 1971 paper by Ruelle and Takens (1971) still proposed Axiom A systems as models for turbulence, but in 1975 Ruelle observed that "Lorenz's work was unfortunately overlooked" (Ruelle 1976a). Guckenheimer and Lanford were among the first people to have shown some interest in this equation (from a mathematical point of view) (Guckenheimer 1976; Lanford 1977). Mathematicians quickly adopted this new object which turned out to be a natural counterexample to Smale's conjecture on the genericity of Axiom A systems. It is impossible to give an exhaustive account of all their work. By 1982 an entire book was devoted to the Lorenz's equation, although it mostly consisted of a list of open problems for mathematicians (Sparrow 1982).

Bowen's review article is interesting at several levels (Bowen, 1978). Smale's theory of Axiom A systems had become solid and, although difficult open questions remained, one had a rather good understanding of their dynamics. A few "dark swans" had appeared in the landscape, like Lorenz's examples, destroying the naïve belief in the genericity of Axiom A systems. However mathematicians were trying to weaken the definition of Axiom A in order to leave space to the newcomer Lorenz. Nowadays, Axiom A systems seem to occupy a much smaller place than one thought at the end of the 1970s. The Axiom A paradigm had to abandon its dominant position... According to (Anosov 2006):

Thus the grandiose hopes of the 1960 s were not confirmed, just as the earlier naive conjectures were not confirmed.

For a more detailed description of the "hyperbolic history" one can also read the introduction of (Hasselblatt 2002), or (Ghys 2010). See also "What is... a horseshoe" by one of the main actors of the field (Shub 2005).

\section{Lorenz's Butterfly as Seen by Mathematicians}

In order to understand Lorenz's butterfly from a mathematical point of view, Guckhenheimer and Williams (1979) introduced a "geometrical model" in 1979. Remember that Lorenz had observed that "his" dynamics seems to be related to the 
iterates of a map $f$ from an interval to itself, even though this interval and this map were only defined "within the limits of accuracy of the printed values". The main idea of Guckenheimer and Williams is to start from a map $f$ of the interval and to construct some vector field in 3-space whose behavior "looks like" the observed behavior of the original Lorenz equation. The question of knowing if the constructed vector field, called the geometric Lorenz model, is actually related to the original Lorenz equation was not considered as important. After all, the original Lorenz equation was a crude approximation of a physical problem and it was unclear whether it was connected with reality, and moreover mathematicians in this group were not really concerned with reality!

The following figure is reprinted from ${ }^{6}$ (Guckenheimer and Williams 1979)

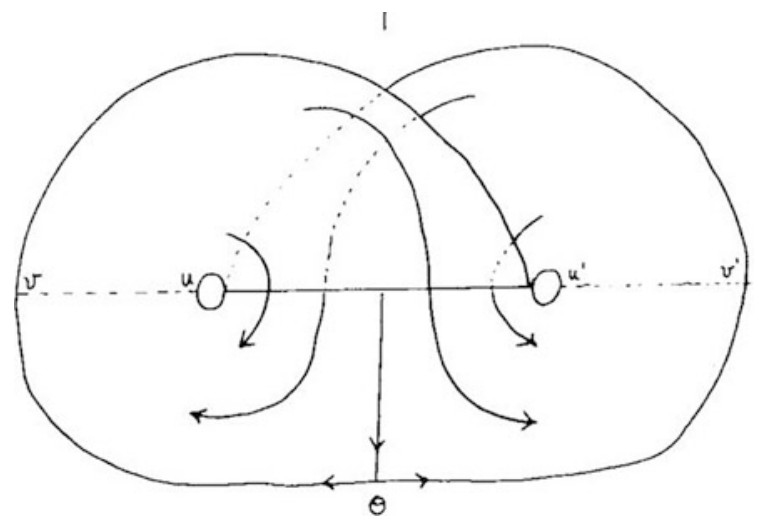

This is a branched surface $\Sigma$ embedded in space. One can define some dynamical system $f^{t}(t \geq 0)$ on $\Sigma$ whose trajectories are sketched on the figure: a point in $\Sigma$ has a future but has no past because of the two leaves which merge along an interval. The first return map on this interval is the given map $f$ from the interval to itself. The dynamics of $f^{t} i$ is easy to understand: the trajectories turn on the surface, either on the left or on the right wing, according to the location of the iterates of the original map $f$. So far, this construction does not yield a vector field. Guckhenheimer and Williams construct a vector field $X(f)$ in some ball $B$ in $\mathbb{R}^{3}$, transversal to the boundary sphere, whose dynamics mimics $f^{t}$. More precisely, denote by $\phi^{t}(x)$ the trajectories of $X(f)$ and by $\Lambda$ the intersection $\cap_{t \geq 0} \phi^{t}(B)$, so that for every point $x$ in $B$, the accumulation points of the trajectory $\phi^{t}(x)$ are contained in $\Lambda$. The vector field $X(f)$ is such that $\Lambda$ is very close to $\Sigma$ and that the trajectories $\phi^{t}(x)$ shadow $f^{t}$. In other words, for every point $x$ in $\Lambda$, there is a point $x^{\prime}$ in $\Sigma$ such that $\phi^{t}(x)$ and $f^{t}\left(x^{\prime}\right)$ stay at a very small distance for all positive times $t \geq 0$. This vector field $X(f)$ is not unique but is well defined up to topological equivalence, i.e. up to some homeomorphism sending trajectories to trajectories. This justifies Lorenz's intuition,

\footnotetext{
${ }^{6}$ Incidentally, this figure shows that the quality of an article does not depend on that of its illustrations.
} 
according to which the attractor $\Lambda$ behaves like a branched surface. Moreover, every vector field in $B$ which is close to $X(f)$ is topologically conjugate to some $X\left(f^{\prime}\right)$ for some map $f^{\prime}$ of the interval which is close to $f$. Furthermore, they construct explicitly a two-parameter family of maps $f_{(a, b)}$ which represent all possible topological equivalence classes. In summary, up to topological equivalence, the vector fields in the neighborhood of $X(f)$ depend on two parameters and are Lorenz like. This is the robustness property mentioned above.

Hence, the open set in the space of vector fields of the form $X(f)$ does not contain any structurally stable vector field. If Smale had known Lorenz's example earlier, he would have saved time! Lorenz's equation does not satisfy Axiom A and cannot be approximated by an Axiom A system. Therefore any theory describing generic dynamical systems should incorporate Lorenz's equation.

As we have mentioned, the geometric models for the Lorenz attractor have been inspired by the original Lorenz equation, but it wasn't clear whether the Lorenz equation indeed behaves like a geometric model. Smale chose this question as one of the "mathematical problems for the next century" in 1998. The problem was positively solved in Tucker (2002). For a brief description of the method used by Tucker, see for instance (Viana 2000).

\section{The Concept of Physical SRB Measures}

\section{Poincaré}

The main method to tackle the sensitivity to initial conditions uses probabilities. This is not a new idea. As mentioned earlier, Laplace realized that solving differential equations requires a "vast intelligence" that we don't have... and suggested developing probability theory in order to get some meaningful information. In his "Science and method", Poincaré gives a much more precise statement. Here is an extract of the chapter on "chance":

When small differences in the causes produce great differences in the effects, why are the effects distributed according to the laws of chance? Suppose a difference of an inch in the cause produces a difference of a mile in the effect. If I am to win in case the integer part of the effect is an even number of miles, my probability of winning will be $1 / 2$. Why is this? Because, in order that it should be so, the integer part of the cause must be an even number of inches. Now, according to all appearance, the probability that the cause will vary between certain limits is proportional to the distance of those limits, provided that distance is very small.

This chapter contains much more information about Poincaré's visionary idea and one can even read some proofs between the lines... In modern terminology, Poincaré considers a vector field $X$ in a ball $B$ in $\mathbb{R}^{n}$, as before. Instead of considering a single point $x$ and trying to describe the limiting behavior of $\phi^{t}(x)$, he suggests choosing some probability distribution $\mu$ in the ball $B$ and to study its 
evolution $\phi_{\star}^{t} \mu$ under the dynamics. He then gives some arguments showing that if $\mu$ has a continuous density, and if there is "a strong sensitivity to initial conditions", the family of measures $\phi_{\star}^{t} \mu$ should converge to some limit $v$ which is independent of the initial distribution $\mu .^{7}$ Even though individual trajectories are sensitive to initial conditions, the asymptotic distribution of trajectories is independent of the initial distribution, assuming that this initial distribution has a continuous density. Amazingly, none of his contemporaries realized that this was a fundamental contribution. This may be due to the fact that Poincaré did not write this idea in a formalized mathematical paper but in a popular book. One would have to wait for about seventy years before this idea could surface again.

\section{Lorenz.}

We have seen that the 1972 conference of Lorenz on the butterfly emphasized the sensitivity to initial conditions and that this idea eventually reached the general public. However, this conference went much further:

More generally, I am proposing that over the years minuscule disturbances neither increase nor decrease the frequency of occurrence of various weather events such as tornados; the most they may do is to modify the sequence in which these events occur.

This is the real message that Lorenz wanted to convey: the statistical description of a dynamical system could be insensitive to initial conditions. Unfortunately, this idea is more complicated to explain and did not become as famous as the "easy" idea of sensitivity to initial conditions.

\section{Sinai, Ruelle, Bowen}

Mathematicians also (re)discovered this idea in the early 1970s, gave precise definitions and proved theorems. A probability measure $v$ in the ball $B$, invariant by $\phi^{t}$, is an SRB measure (for Sinai-Ruelle-Bowen), also called a physical measure, if, for each continuous function $u: B \rightarrow \mathbb{R}$, the set of points $x$ such that

$$
\lim _{T \rightarrow \infty} \frac{1}{T} \int_{0}^{T} u\left(\phi^{t}(x)\right) d t=\int_{B} u d v
$$

\footnotetext{
7 I may be exaggerating because of my excessive worship of Poincaré, but it seems to me that, in modern terminology, Poincaré explains that the limiting probability $v$ is absolutely continuous on instable manifolds and may not be continuous on stable manifolds.
} 
has nonzero Lebesgue measure. This set of points is called the basin of $v$ and denoted by $B(v)$. Sinai, Ruelle and Bowen (Sinai 1972; Ruelle 1976b; Bowen 1978) proved that this concept is indeed relevant in the case of Axiom A dynamics. If $X$ is such a vector field in some ball $B$, there is a finite number of SRB measures $v_{1}, \ldots, v_{k}$ such that the corresponding basins $B\left(v_{1}\right), \ldots, B\left(v_{k}\right)$ cover $B$, up to a Lebesgue negligible set. Of course, the proof of this important theorem is far from easy but its general structure follows the lines sketched in Poincaré paper...

In summary, the existence of SRB measures is the right answer to the "malediction" of the sensitivity to initial conditions. In the words of Lorenz, "the frequency of occurrence of various weather events such as tornados" could be insensitive to initial conditions. If for example the ball $B$ represents the phase space of the atmosphere and $u: B \rightarrow \mathbb{R}$ denotes the temperature at a specific point on the Earth, the average $\frac{1}{T} \int_{0}^{T} u\left(\phi^{t}(x)\right) d t$ simply represents the average temperature in the time interval $[0, T]$. If there is an SRB measure, this average converges to $\int u d v$, independently of the initial position $x$ (at least in the basin of $v$ ). The task of the forecaster changed radically: instead of guessing the position of $\phi^{t}(x)$ for a large $t$, he or she tries to estimate an SRB measure. This is a positive statement about chaos as it gives a new way of understanding the word "prevision". It is unfortunate that such an important idea did not reach the general population. Poor Brazilian butterflies! They are now unable to change the fate of the world!

The quest for the weakest conditions that guarantee the existence of SRB measures is summarized in the book (Bonatti et al. 2005). This question is fundamental since, as we will see, one hopes that "almost all" dynamical systems admit SRB measures.

The geometric Lorenz models are not Axiom A systems, hence are not covered by the works of Sinai, Ruelle and Bowen. However, it turns out that the Lorenz attractor supports a unique SRB measure (Bunimovich 1983; Pesin 1992). Lorenz was right!

\section{Palis}

The history of dynamical systems seems to be a long sequence of hopes... quickly abandoned. A non chaotic world, replaced by a world consisting of Axiom A systems, in turn destroyed by an abundance of examples like Lorenz's model. Yet, mathematicians are usually optimists, and they do not hesitate to remodel the world according to their present dreams, hoping that their view will not become obsolete too soon. Palis $(1995,2005,2008)$ proposed such a vision in a series of three articles. He formulated a set of conjectures describing the dynamics of "almost all" vector fields. These conjectures are necessarily technical, and it would not be useful to describe them in detail here. I will only sketch their general spirit.

The first difficulty — which is not specific to this domain - is to give a meaning to "almost all" dynamics. The initial idea from the 1960s was to describe an open 
dense set in the space of dynamical systems, or at least, a countable intersection of open dense sets, in order to use Baire genericity. Yet, this notion has proved to be too strict. Palis uses a concept of "prevalence" whose definition is technical but which is close in spirit to the concept of "full Lebesgue measure". Palis finiteness conjecture asserts that in the space of vector fields on a given ball $B$, the existence of a finite number of SRB measures whose basins cover almost all the ball is a prevalent property.

Currently, the Lorenz attractor serves as a model displaying phenomena that are believed be characteristic of "typical chaos", at least in the framework of mathematical chaos. Even so, the relevance of the Lorenz model to describe meteorological phenomena remains largely open (Robert 2001).

\section{Communicating Mathematical Ideas?}

In Poincaré's time, the total number of research mathematicians in the world was probably of the order of 500. Even in such a small world, even with the expository talent of Poincaré as a writer, we have seen that some important ideas could not reach the scientific community. The transmission of ideas in the theory of chaos, from Poincaré to Palis has not been efficient. In the 1960s we have seen that the Lorenz equation took ten years to cross America from the east coast to the west coast, and from physics to mathematics. Of course, the number of scientists had increased a lot. In our 21 st century, the size of the mathematical community is even bigger ( $\sim 50,000$ research mathematicians?) and the physical community is much bigger. Nowadays, the risk is not only that a good idea could take ten years to go from physics to mathematics: there could be tiny subdomains of mathematics that do not communicate at all. Indeed, very specialized parts of mathematics that look tiny for outsiders turn out to be of a respectable size, say of the order of 500, and can transform into "scientific bubbles". As Lovász (2006) writes in his "Trends in Mathematics: How they could Change Education?":

A larger structure is never just a scaled-up version of the smaller. In larger and more complex animals an increasingly large fraction of the body is devoted to 'overhead': the transportation of material and the coordination of the function of various parts. In larger and more complex societies an increasingly large fraction of the resources is devoted to nonproductive activities like transportation information processing, education or recreation. We have to realize and accept that a larger and larger part of our mathematical activity will be devoted to communication.

Of course, this comment does not only apply to mathematics but to Science in general and to the society at large. Nowadays, very few university curricula include courses on communication aimed at mathematicians. We need to train mediators who can transport information at all levels. Some will be able to connect two different areas of mathematics, some will link mathematics and other sciences, and some others will be able to communicate with the general public. It is important that we consider this kind of activity as a genuine part of scientific research and that it 
could attract our most talented students, at an early stage of their career. We should not only rely on journalists for this task and we should prepare some of our colleagues for this noble purpose. We have to work together and to improve mathematical communication. We should never forget that a mathematical giant like Poincaré took very seriously his popular essays and books, written for many different audiences.

Open Access This chapter is distributed under the terms of the Creative Commons Attribution Noncommercial License, which permits any noncommercial use, distribution, and reproduction in any medium, provided the original author(s) and source are credited.

\section{References}

Abraham, R. \& Smale, S. (1968). Nongenericity of $\Omega$-stability. Global Analysis (Proc. Sympos. Pure Math., Vol. XIV, Berkeley, Calif., 1968), 5-8. Amer. Math. Soc., Providence.

Andronov, A., \& Pontrjagin, L. (1937). Systèmes grossiers. Dokl. Akad. Nauk SSSR, 14.

Anosov, D.V. (1962). Roughness of geodesic flows on compact Riemannian manifolds of negative curvature. Dokl. Akad. Nauk SSSR, 145, 707-709.

Anosov, D.V. (2006). Dynamical systems in the 1960s: the hyperbolic revolution. In Mathematical events of the twentieth century, 1-17, Springer, Berlin.

Aubin D., \& Dahan Dalmedico A. (2002). Writing the history of dynamical systems and chaos: longue durée and revolution, disciplines and cultures. Historia Math. 29(3), 273-339.

Birkhoff, G.D. (1927). Dynamical Systems. vol. 9 of the American Mathematical Society Colloquium Publications (Providence, Rhode Island: American Mathematical Society).

Bonatti, C., Diaz, L., \& Viana. M. (2005). Dynamics beyond uniform hyperbolicity. A global geometric and probabilistic perspective. Encyclopaedia of Mathematical Sciences, (102). Mathematical Physics, III. Springer-Verlag, Berlin.

Bowen, R. (1978). On Axiom A diffeomorphisms. Regional Conference Series in Mathematics, 35. American Mathematical Society, Providence, R.I.

Bunimovich, L.A. (1983). Statistical properties of Lorenz attractors. Nonlinear dynamics and turbulence, Pitman, 7192.

Duhem, P. (1906). La théorie physique; son objet, sa structure. English transl. by P.P. Wiener, The aim and structure of physical theory, Princeton University Press, 1954.

Ghys, E. (2010). L'attracteur de Lorenz, paradigme du chaos. Séminaire Poincaré, XIV.

Gleick, J. (1987). Chaos: Making a New Science. Viking Penguin.

Guckenheimer, J. (1976). A strange, strange attractor. In The Hopf Bifurcation, Marsden and McCracken, eds. Appl. Math. Sci., Springer-Verlag.

Guckenheimer, J., \& Williams, R.F. (1979). Structural stability of Lorenz attractors. Inst. Hautes Études Sci. Publ. Math., 50, 59-72.

Hadamard, J. (1898). Les surfaces à courbures opposées et leurs lignes géodésiques. Journal de mathématiques pures et appliqués, 5e série 4, 27-74.

Hasselblatt, B. (2002). Hyperbolic dynamical systems. Handbook of dynamical systems, Vol. 1A, 239-319, North-Holland, Amsterdam.

Kahane, J.-P. (2008). Hasard et déterminisme chez Laplace. Les Cahiers Rationalistes, 593.

Lanford, O. (1977). An introduction to the Lorenz system. In Papers from the Duke Turbulence Conference (Duke Univ., Durham, N.C., 1976), Paper No. 4, i + 21 pp. Duke Univ. Math. Ser., Vol. III, Duke Univ., Durham, N.C.

Laplace, P.S. (1814). Essai philosophique sur les probabilités. English transl. by A.I. Dale, Philosophical essay on probabilities, Springer, 1995. 
Lorenz, E.N. (1960). Maximum simplification of the dynamic equations. Tellus, 12, 243-254.

Lorenz, E.N. (1962). The statistical prediction of solutions of dynamic equations. Proc. Internat. Sympos. Numerical Weather Prediction, Tokyo, 629-635.

Lorenz, E.N. (1963). Deterministic non periodic flow. J. Atmosph. Sci., 20, 130-141.

Lorenz, E.N. (1972). Predictability: does the flap of a butterfly's wings in Brazil set off a tornado in Texas? 139th Annual Meeting of the American Association for the Advancement of Science (29 Dec 1972), in Essence of Chaos (1995), Appendix 1, 181.

Lorenz, E.N. (1991). A scientist by choice. Kyoto Award lecture. (available at http://eaps4.mit. edu/research/Lorenz/Miscellaneous/Scientist_by_Choice.pdf)

Lovász, L. (2006). Trends in Mathematics: How they could Change Education? In The Future of Mathematics Education in Europe, Lisbon.

Maxwell, J.C. (1876). Matter and Motion. New ed. Dover (1952).

Palis, J. (1995). A global view of dynamics and a conjecture on the denseness of finitude of attractors. Géométrie complexe et systèmes dynamiques (Orsay, 1995). Astérisque, 261 (2000), xiii-xiv, 335-347.

Palis, J. (2005). A global perspective for non-conservative dynamics. Ann. Inst. H. Poincaré Anal. Non Linéaire, 22(4), 485-507.

Palis, J. (2008). Open questions leading to a global perspective in dynamics. Nonlinearity, 21(4), T37-T43.

Pesin, Y. (1992). Dynamical Systems With Generalized Hyperbolic Attractors: Hyperbolic, Ergodic and Topological Properties, Ergod. Theory and Dyn. Syst., 12:1 (1992) 123-152.

Poincaré, H. (1881). Mémoire sur les courbes définies par une équation différentielle. Journal de mathématiques pures et appliqués, 7, 375-422.

Poincaré, H. (1890). Sur le problème des trois corps et les équations de la dynamique. Acta Mathematica, 13, 1-270.

Poincaré, H. (1908). Science et méthode. Flammarion. English transl. by F. Maitland, Science and Method, T. Nelson and Sons, London, 1914.

Robert, R. (2001). L'effet papillon n'existe plus ! Gaz. Math., 90, 11-25.

Ruelle, D. (1976a). The Lorenz attractor and the problem of turbulence. In Turbulence and NavierStokes equations (Proc. Conf., Univ. Paris-Sud, Orsay, 1975), 146-158, Lecture Notes in Math., 565, Springer, Berlin, (1976).

Ruelle, D. (1976b). A measure associated with axiom-A attractors. Amer. J. Math., 98(3), 619-654.

Ruelle, D., \& Takens, F. (1971). On the nature of turbulence. Comm. Math. Phys., 20, 167-192.

Shub, M. (2005). What is... a horseshoe?. Notices Amer. Math. Soc., 52(5), 516-517.

Sinai, Ja. G. (1972). Gibbs measures in ergodic theory, (in Russian). Uspehi Mat. Nauk., 27(4), 21-64.

Smale, S. (1960). On dynamical systems. Bol. Soc. Mat. Mexicana, 5, 195-198.

Smale, S. (1961). A structurally stable differentiable homeomorphism with an infinite number of periodic points. Qualitative methods in the theory of non-linear vibrations (Proc. Internat. Sympos. Non-linear Vibrations, Vol. II, 1961) Izdat. Akad. Nauk Ukrain. SSR, Kiev, 365-366.

Smale, S. (1966). Structurally stable systems are not dense. Amer. J. Math., 88, 491-496.

Smale, S. (1967). Differentiable dynamical systems. Bull. Amer. Math. Soc., 73, 747-817.

Smale, S. (1998). Finding a horseshoe on the beaches of Rio. Math. Intelligencer, 20 (1), 39-44.

Smale, S. (1998b). Mathematical problems for the next century. Math. Intelligencer, 20(2), 7-15.

Sparrow, C. (1982). The Lorenz equations: bifurcations, chaos, and strange attractors. Applied Mathematical Sciences, 41. Springer-Verlag, New York-Berlin.

Tucker, W. (2002). A rigorous ODE solver and Smale's 14th problem. Found. Comput. Math., 2 (1), 53-117.

Viana, M. (2000). What's new on Lorenz strange attractors? Math. Intelligencer, 22(3), 6-19.

Williams, R.F. (1979). The structure of Lorenz attractors. Inst. Hautes Études Sci. Publ. Math., 50, 73-99. 